\title{
A comunidade vegetal e as características abióticas de um campo de murundu em Uberlândia, MG
}

\author{
Isa Lucia de Morais Resende ${ }^{1}$, Glein Monteiro de Araújo ${ }^{1,3}$, Ana Paula de Assis Oliveira ${ }^{1}$, \\ Ana Paula de Oliveira ${ }^{2}$ e Rubem Samuel de Ávila Júnior ${ }^{1}$
}

Recebido em 25/02/2003. Aceito em 15/06/2003

\begin{abstract}
RESUMO - (A comunidade vegetal e as características abióticas de um campo de murundu em Uberlândia, MG). Murundu é um tipo de microrrelevo em forma de pequena elevação, geralmente arredondado, muitas vezes apresentando solo e vegetação diferentes da área circundante. Todos os morrotes encontrados em 1,08ha de um campo de murundu foram medidos (comprimento, largura e altura). Determinouse a profundidade do lençol freático e as características do solo sobre os mesmos e na área plana adjacente. A vegetação lenhosa (cipós, arbustos e árvores) com diâmetro do caule ao nível do solo $\geq 5 \mathrm{~mm}$ foi amostrada em 48 morrotes. Encontraram-se 434 elevações (31,4\% da área) das quais $24,3 \%$ apresentaram vegetação lenhosa. A profundidade do lençol freático sob os murundus com vegetação lenhosa variou de 99,2 a 206,8cm. O solo estudado apresentou baixo teor de bases trocáveis e pequena diferença na disponibilidade de nutrientes entre os murundus e a área plana próxima. Foram amostrados 116 espécies, 72 gêneros e 42 famílias. Matayba guianensis Aubl. e Erythroxylum suberosum A. St.-Hil. apresentaram maiores densidades e Copaifera langsdorffii Desf. e Blepharocalyx salicifolius (Kunth) O. Berg., maiores áreas basais. A maior diversidade em relação a outros trabalhos pode ser decorrente do pisoteio, pastejo bovino e queimadas.
\end{abstract}

Palavras-chave: cerrado, murundu, vegetação, lençol freático

ABSTRACT - (Plant community and abiotic characteristics of a murundu field in Uberlândia, MG). Murundus are small usually round mounds, that often present soil and vegetation different from the surrounding level area. All micro-elevations incountered in a 1.08ha murundu field had their length, width and height measured. The depth of the water table was measured and soil characteristics were obtained within and outside the murundus. All woody plants (lianas, trees, and shrubs) with a diameter of $\geq 5 \mathrm{~mm}$ at ground level were identified. A total 434 elevations (31.4\% of the total area) were encontered, of which 48 (24.3\%) contained woody plants. The mound vegetation comprised 116 species belonging to 72 genera, and 42 families. The depth of the water table for those murunduns with woody plants varied between 99.2 and $206.8 \mathrm{~cm}$. The studied soil showed a low content of exchangeable bases, but only slight differences were observed between murundus and level soils. Matayba guianensis Aubl. and Erythroxylum suberosum A. St.-Hil. had the highest densities, while Copaifera langsdorffii Desf. and Blepharocalyx salicifolius (Kunth) O. Berg. showed the highest basal areas. The heigher diversity encountered here in comparison to other published reports may be due to cattle grazing and trampling as well as burning.

Key words: cerrado, earthmounds, vegetation, water table

\section{Introdução}

O Brasil possui cinco grandes tipos de províncias vegetacionais - Floresta Amazônica do Norte, Cerrado do Brasil Central, Caatinga do Nordeste, Floresta Atlântica e as Pradarias de campo limpo graminoso do Sul. Em cada província, o tipo principal cobre de 75 a $90 \%$ da área, sendo o restante ocupado por outros tipos de vegetações terrestres ou brejosas, determinadas por condições especiais do substrato, como por exemplo, o campo de murundu (Eiten 1990).

O campo de murundu é constituído por uma área plana, inundável no período das chuvas, onde se encontram inúmeros morrotes. A área plana e os murundus menores são cobertos por vegetação campestre e os maiores, por vegetação lenhosa do Cerrado (Eiten 1985; Diniz de Araújo Neto et al. 1986; Oliveira-Filho \& Furley 1990; Oliveira-Filho 1992a). A atividade de térmitas, juntamente com processos erosivos, parecem moldá-los a uma formação arredondada ou elíptica, apresentando altura máxima de um a dois metros (Eiten 1985; Diniz de Araújo Neto et al. 1986; Oliveira-Filho 1992a; Ponce \& Cunha 1993). Na região do Cerrado, sítios com essa característica ocorrem nas planícies de inundação de alguns rios (Oliveira-Filho \& Furley 1990; Schneider \& Silva 1991) e entre o campo-cerrado e campo-úmido e/ou mata de galeria (Eiten 1972; Diniz de Araújo Neto et al. 1986;

\footnotetext{
1 Instituto de Biologia, Universidade Federal de Uberlândia, Campus Umuarama, CEP 38400-902, Uberlândia, MG, Brasil

2 Departamento de Ecologia, Universidade de Brasília, CEP 70719-970, Brasília, DF, Brasil

3 Autor para correspondência: glein@ufu.br
} 
Furley 1986). No Pantanal do Mato Grosso, esse ambiente "encalombado" pode ser encontrado nas áreas de transição com o Cerrado (Eiten 1985; Oliveira-Filho \& Furley 1990).

Sabe-se que a maior parte da vegetação lenhosa que ocorre nos murundus pertence às espécies de Cerrado (Eiten 1985; Furley \& Ratter 1988; OliveiraFilho \& Furley 1990), mas conhece-se apenas um trabalho publicado (Oliveira-Filho 1992b) que determinou a sua composição florística, densidade e dominância, em um sítio no Pantanal do Mato Grosso. Nesse sentido, visando ampliar o conhecimento sobre as comunidades vegetais lenhosas que ocorrem nesses ambientes, o presente estudo teve como objetivo determinar a diversidade, densidade e dominância de espécies lenhosas de um campo de murundu, na região do Cerrado, e relacioná-las com as características ambientais onde se encontram.

\section{Material e métodos}

O campo de murundu onde foi realizado o trabalho localiza-se próximo à rodovia BR 452 (195'9''S e $48^{\circ} 2$ ' 14'O), na Fazenda Munjolinho, cerca de $43 \mathrm{~km}$ do centro da cidade de Uberlândia, MG. É uma área de aproximadamente 15 ha e situa-se próxima ao rio
Uberabinha. A topografia do local é plana (declividade de 1 a $2 \%$ ) e no período das chuvas formam-se pequenas lagoas e/ou brejos em áreas de depressões menores. No sítio predomina a vegetação herbácea (principalmente Cyperaceae e Poaceae) na área plana e nos murundus pequenos; nos maiores ocorrem também arbustos e árvores. Na figura 1 encontra-se uma vista parcial da área, mostrando, em primeiro plano, um murundu com maior volume de solo sendo ocupado com árvores, arbustos e ervas. O campo de murundu é utilizado como pastagem e, no período mais seco, é eventualmente queimado.

O clima da região, de acordo com a classificação de Köppen, é do tipo Aw megatérmico, com chuvas no verão e estação relativamente seca no inverno. Os meses com maior precipitação pluviométrica são dezembro e janeiro, e as menores ocorrem em junho e julho (Rosa et al. 1991).

$\mathrm{Na}$ chapada Uberlândia-Uberaba ocorrem depressões suaves com topografias planas ocupadas por morrotes de diversos tamanhos. Esses locais denominados na região como covoais e/ou varjões apresentam solos de origem hidromórfica, com textura argilosa, do tipo caulinita e gipsita. A análise micromorfológica nos murundus mostra a importância da atividade biológica nos solos desses locais (Schneider

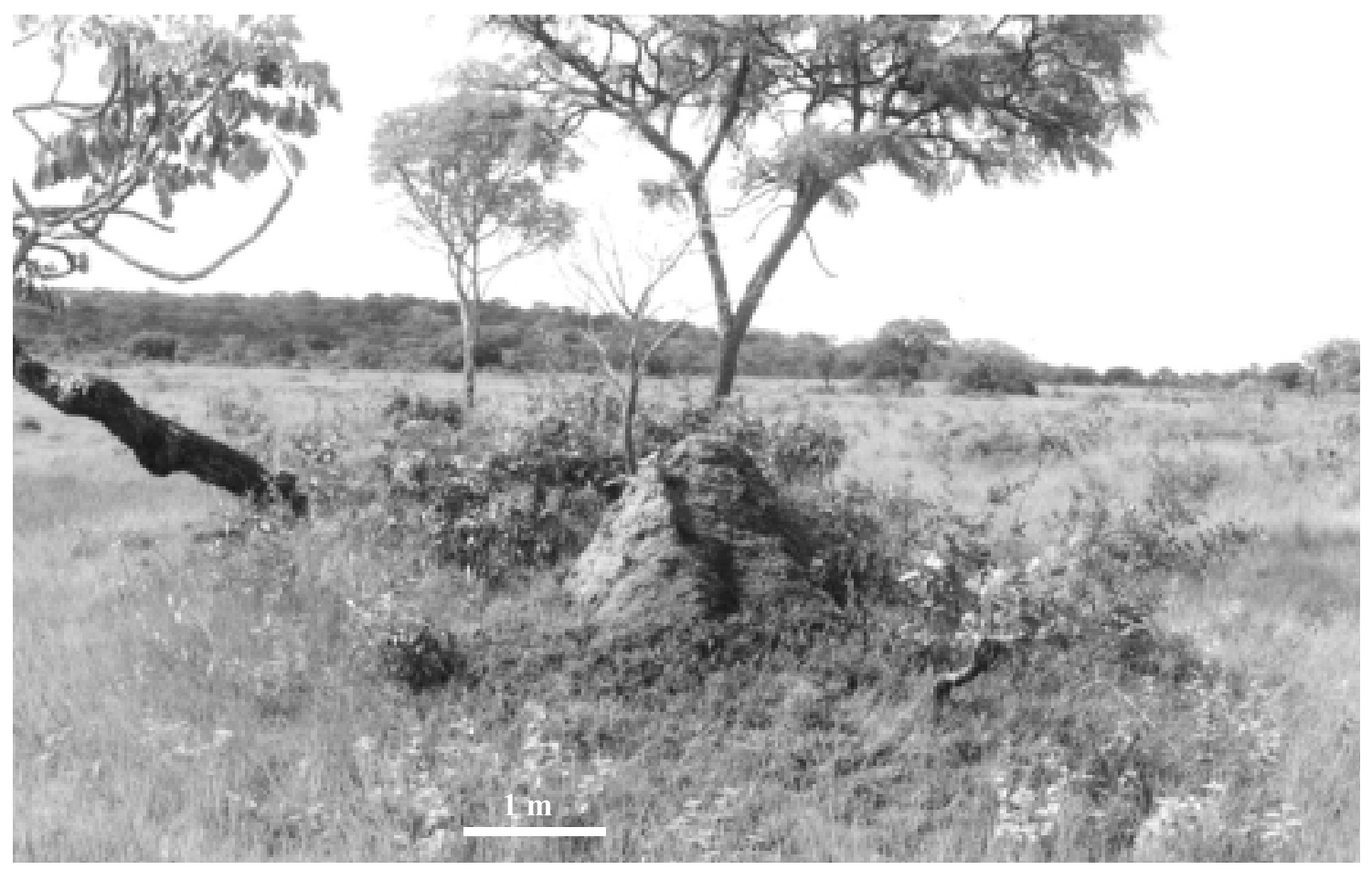

Figura 1. Aspecto do campo de murundu estudado em Uberlândia, MG, tendo em primeiro plano um morrote com maior volume de solo ocupado por vegetação. 
\& Silva 1991). Sítios semelhantes a esses podem ser encontrados nas planícies de inundação dos rios Araguaia, Tocantins, Paraguai e Cuiabá (Oliveira-Filho \& Furley 1990).

O comprimento, a largura e a altura de todos os murundus que ocorreram em 1,08ha da área amostrada (12 parcelas de $30 \mathrm{~m} \times 30 \mathrm{~m}$ ) foram medidos e classificados em dois tipos: aqueles que só apresentavam vegetação herbácea e os que possuíam herbácea, arbustiva e arbórea. Calculou-se a área e o volume de cada murundu, considerando sua forma semi-elipsóide, pelas fórmulas: Área $=\pi / 4 \times$ compr. $\times$ larg. e Volume $=\pi / 6 \times$ compr. $\times$ larg. $\times$ alt. (Oliveira-Filho 1992b). Com os dados de área e volume, foram construídos gráficos de distribuição de frequiência abrangendo os dois tipos de murundus.

Para verificar a profundidade do lençol freático, de setembro (final da estação relativamente seca) a fevereiro (estação chuvosa) realizaram-se perfurações no solo sobre os murundus que tinham vegetação lenhosa e em áreas planas, em suas proximidades. As perfurações foram feitas na parte central mais alta de seis murundus e em seis locais planos, em suas proximidades, utilizando-se um trado holandês, com cinco centímetros de diâmetro. A profundidade do lençol freático foi determinada com trena metálica.

Para caracterizar o solo quanto ao teor de matéria orgânica e disponibilidade de nutrientes foram retiradas seis amostras compostas de solo (0 a $10 \mathrm{~cm}$ de profundidade), sendo três oriundas de murundus que apresentavam vegetação lenhosa, e as demais em locais planos cobertos por vegetação herbácea. As análises foram feitas no Laboratório de Análise de Solos e Calcáreos da Universidade Federal de Uberlândia (UFU), de acordo com a metodologia preconizada pela EMBRAPA (1979).

A amostragem da vegetação foi realizada nos 48 murundus ocupados por espécies lenhosas situados em 12 parcelas de $30 \mathrm{~m} \times 30 \mathrm{~m}$, plotadas sistematicamente na área. Os indivíduos lenhosos (cipós, arbustos e árvores) amostrados tinham diâmetro do caule $\geq 5 \mathrm{~mm}$ ao nível do solo. Nas espécies ramificadas, mediram-se os três perfílios com maior diâmetro. Cada espécie foi classificada quanto ao hábito, de acordo com o porte da planta, conforme Bernacci \& Leitão-Filho (1996) e Rizzini \& Rizzini (1983). A identificação das espécies foi feita no local da amostragem e/ou por comparação com exsicatas existentes no acervo do Herbário HUFU (Herbarium Uberlandensis) da Universidade Federal de Uberlândia e consulta a especialistas.
A densidade e dominância absoluta de cada espécie nos murundus amostrados e os Índices de Diversidade de Shannon (H') e de Eqüabilidade (E) foram calculados utilizando-se fórmulas usuais (Cain \& Castro 1959; Matteucci \& Colma 1982).

\section{Resultados}

Em 1,08ha de campo de murundu encontraram-se 434 morrotes com altura média de $0,29 \mathrm{~m}$, ocupando $31,4 \%$ da área. Os murundus com vegetação exclusivamente herbácea (386) ocuparam 7,1\% da área. Destes, $71 \%$ apresentaram menos de $2,3 \mathrm{~m}^{2}$ de área e $78,5 \%$ de volume menor que $0,47 \mathrm{~m}^{3}$ (Fig. 2). Os demais (48) com herbáceas, arbustos e árvores ocuparam 24,3\% do sítio sendo encontradas áreas mínima, média e máxima de $3,3 \mathrm{~m}^{2}, 54,6 \mathrm{~m}^{2} \mathrm{e} 152,6 \mathrm{~m}^{2} \mathrm{e}$ volumes de $1,08 \mathrm{~m}^{3}$, $32,5 \mathrm{~m}^{3} \mathrm{e} 82,3 \mathrm{~m}^{3}$, respectivamente. Entre estes murundus $(85,4 \%)$ predominaram valores de área até $83,3 \mathrm{~m}^{2} \mathrm{e} \mathrm{com}$ volume inferior a $50,4 \mathrm{~m}^{3}(79,2 \%)$ (Fig. 3).

A profundidade média do lençol freático, sob os murundus com vegetação lenhosa, mesmo no período de chuvas, ocorreu abaixo de $0,99 \mathrm{~m}$ em relação à superfície do solo. Na parte plana, coberta por herbáceas, apenas em outubro, final da estação seca, o lençol freático ocorreu acima de $1 \mathrm{~m}$ de profundidade (Fig. 4).
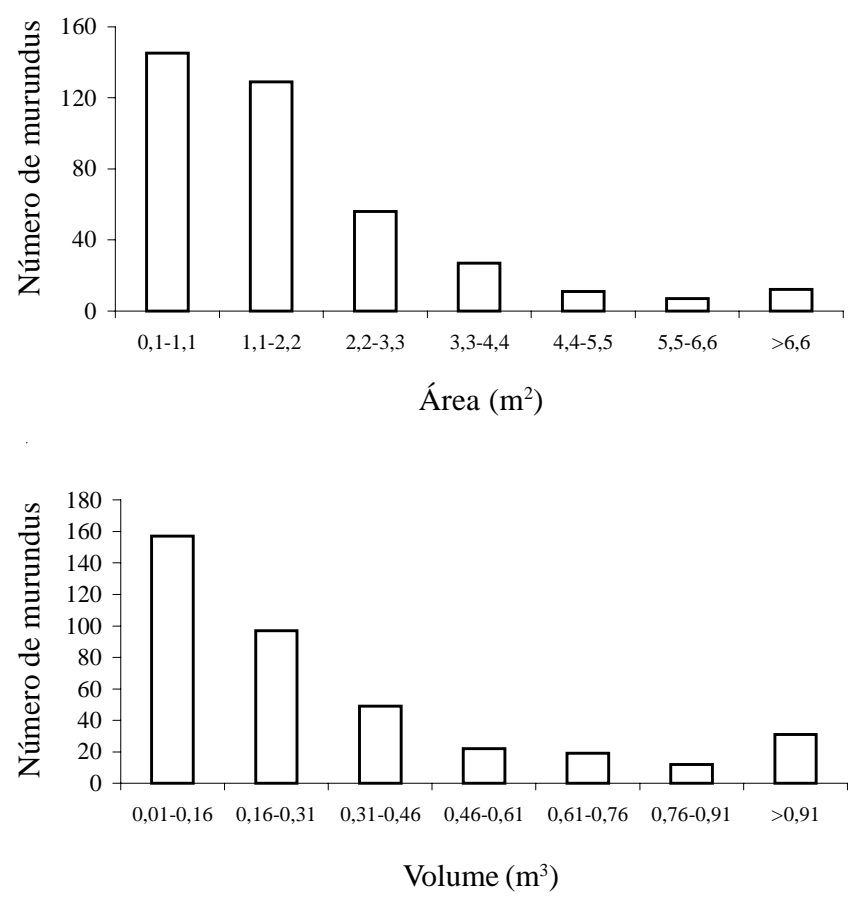

Figura 2. Distribuição de frequiência da área e do volume dos murundus, ocupados por vegetação herbácea, na Fazenda Munjolinho, em Uberlândia, MG. 


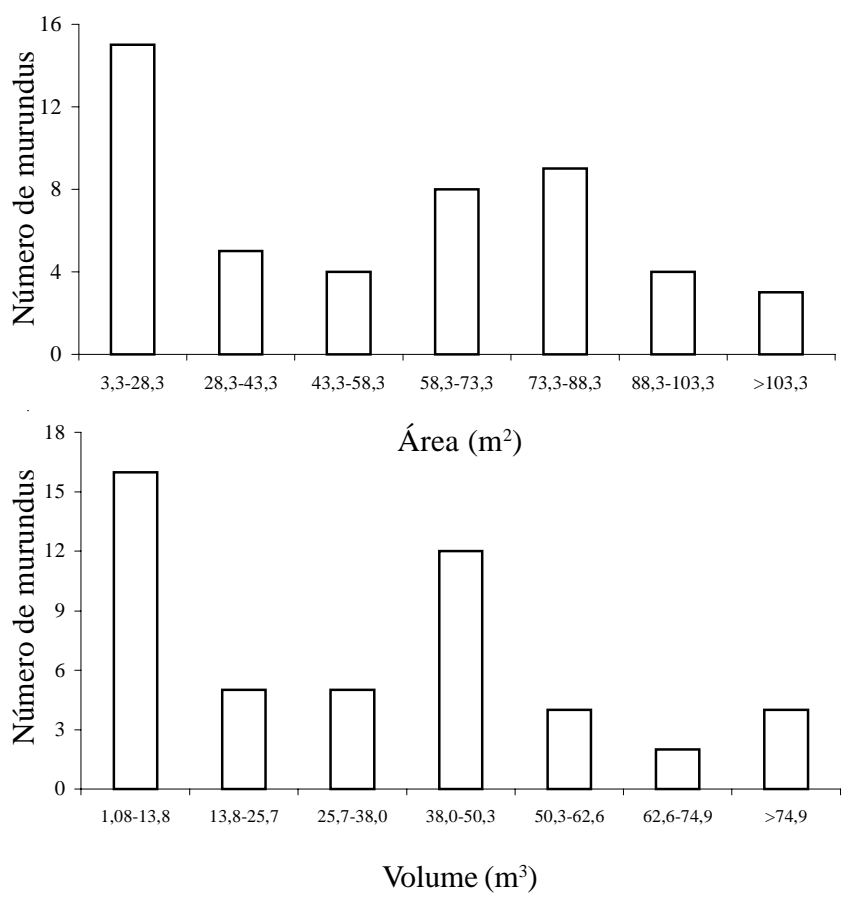

Figura 3. Distribuição de freqüência da área e volume dos 48 murundus, ocupados por vegetação herbácea e lenhosa na Fazenda Munjolinho, em Uberlândia, MG.

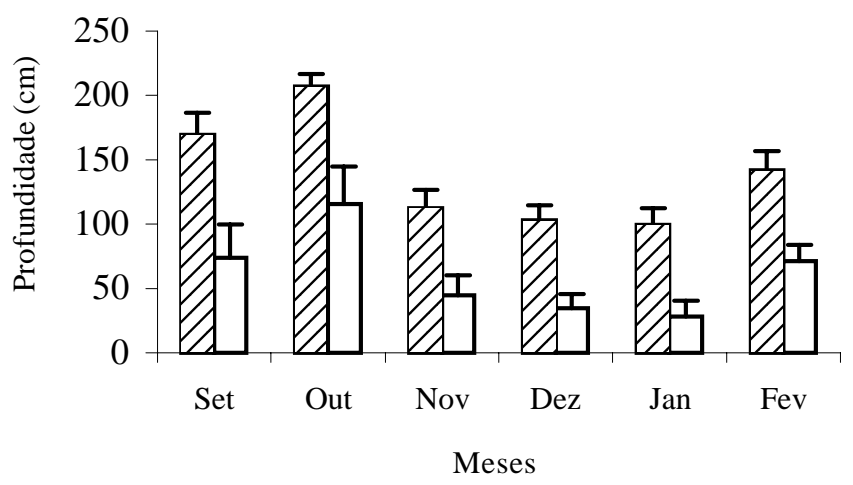

Figura 4. Profundidade média do lençol freático e respectivo desvio padrão de setembro a fevereiro na área de murundu em Uberlândia, MG (n=6). $\square$ Sob o murundu; $\square$ fora do murundu.
O solo da área estudada (Tab. 1) caracterizou-se pela baixa disponibilidade de bases trocáveis e alto teor de $\mathrm{Al}$ disponível. Nos murundus que apresentaram vegetação herbácea e lenhosa foram encontrados maiores teores de $\mathrm{K}, \mathrm{Ca}$ e $\mathrm{Mg}$ e, na área plana adjacente houve maior disponibilidade de $\mathrm{P}$ e $\mathrm{K}$.

Nos 48 murundus com vegetação lenhosa, amostraram-se 2.470 indivíduos pertencentes a 116 espécies, 72 gêneros e 42 famílias. A comunidade vegetal arbustiva representou 57,8\% das espécies e $60,9 \%$ dos indivíduos amostrados nos murundus (Tab. 2). Em geral, quanto maior o volume do murundu, maior o número de espécies, de indivíduos e a área basal. Myrtaceae foi a família com maior número de espécies (19) que, juntamente com nove outras mais ricas, perfizeram 63,8\% do total amostrado (Fig. 5).

As dez espécies com maior área basal representaram somente $36,5 \%$ do número de indivíduos amostrados, sendo as duas arbustivas, Matayba guianensis (555) e Erythroxylum suberosum (217), as que apresentaram as maiores densidades. As dez espécies com maior dominância somam $65,2 \%$ do valor total. As espécies arbóreas Copaifera langsdorffii $\left(1.817,1 \mathrm{~m}^{2}\right.$ de madeira) e Blepharocalyx salicifolius $\left(1.091,1 \mathrm{~m}^{2}\right)$ foram as que tiveram os maiores valores de dominância (Tab. 2).

O Índice de Diversidade de Shannon ( $\left.\mathrm{H}^{\prime}\right)$ para espécie foi de 3,56 nats/indivíduo e a Equabilidade, de 0,75 .

\section{Discussão}

A proporção da área ocupada pelos murundus, nesse trabalho, está entre os valores encontrados por Furley (1986) e Diniz de Araújo Neto et al. (1986) no Distrito Federal (10-50\%), porém maior do que os determinados por Oliveira-Filho (1992a), na Baixada Cuiabana, e Eiten (1985), no Pantanal do Mato Grosso

Tabela 1. Características do solo nos murundus com vegetação herbácea e lenhosa e na área plana na Fazenda Munjolinho, Uberlândia, MG (n = 3), (t) CTC efetiva, (V) saturação por bases, (m) saturação por Al, (MO) matéria orgânica. Desvio padrão entre parênteses.

\begin{tabular}{|c|c|c|c|c|c|c|c|c|c|c|}
\hline \multirow{2}{*}{ Localização } & \multirow{2}{*}{$\mathrm{pH}$} & $\mathrm{P}$ & K & $\mathrm{Ca}$ & $\mathrm{Mg}$ & $\mathrm{Al}$ & $\mathrm{t}$ & V & $\mathrm{m}$ & \multirow{2}{*}{$\begin{array}{c}\text { MO } \\
\text { dag.kg }\end{array}$} \\
\hline & & \multicolumn{2}{|c|}{$\mathrm{mg} \cdot \mathrm{dm}^{-3}$} & \multicolumn{4}{|c|}{$\operatorname{cmol}(+) \cdot \mathrm{dm}^{-3}$} & \multicolumn{2}{|c|}{$\%$} & \\
\hline Murundu & $\begin{array}{c}4,4 \\
(0,3)\end{array}$ & $\begin{array}{c}0,13 \\
(0,06)\end{array}$ & $\begin{array}{c}32,3 \\
(24,9)\end{array}$ & $\begin{array}{c}0,33 \\
(0,40)\end{array}$ & $\begin{array}{c}0,03 \\
(0,06)\end{array}$ & $\begin{array}{c}0,57 \\
(0,11)\end{array}$ & $\begin{array}{c}1,20 \\
(0,59)\end{array}$ & $\begin{array}{c}6,44 \\
(3,65)\end{array}$ & $\begin{array}{c}66,70 \\
(14,3)\end{array}$ & $\begin{array}{c}4,00 \\
(0,89)\end{array}$ \\
\hline Área plana & $\begin{array}{c}4,2 \\
(0,1)\end{array}$ & $\begin{array}{c}0,43 \\
(0,49)\end{array}$ & $\begin{array}{l}38,6 \\
(5,5)\end{array}$ & $\begin{array}{c}0,03 \\
(0,06)\end{array}$ & - & $\begin{array}{c}1,73 \\
(0,15)\end{array}$ & $\begin{array}{c}1,52 \\
(0,61)\end{array}$ & $\begin{array}{c}1,66 \\
(0,79)\end{array}$ & $\begin{array}{l}93,00 \\
(2,28)\end{array}$ & $\begin{array}{c}4,80 \\
(0,23)\end{array}$ \\
\hline
\end{tabular}


Tabela 2. Espécies, em ordem de dominância absoluta, amostradas no campo de murundu da Fazenda Munjolinho, Uberlândia, MG. $\mathrm{H}=$ hábito de vida (Arv = arbóreo, Arb = arbustivo, Cip = cipó); DA = Densidade absoluta (número de indivíduos/48 murundus); DoA = Dominância absoluta (área basal $\mathrm{em} \mathrm{cm}^{2} / 48$ murundus).

\begin{tabular}{|c|c|c|c|}
\hline Famílias/Espécies & $\mathrm{H}$ & DA & DoA \\
\hline \multicolumn{4}{|l|}{ LEGUMINOSAE } \\
\hline $\begin{array}{l}\text { Copaifera langsdorffii Desf. } \\
\text { MYRTACEAE }\end{array}$ & Arv & 4 & $1.817,09$ \\
\hline $\begin{array}{l}\text { Blepharocalyx salicifolius (Kunth) } \\
\text { O. Berg. }\end{array}$ & Arv & 10 & $1.816,60$ \\
\hline ERYTHROXYLACEAE & & & \\
\hline $\begin{array}{l}\text { Erythroxylum suberosum A. St.-Hil. } \\
\text { BOMBACACEAE }\end{array}$ & Arb & 217 & $1.091,05$ \\
\hline $\begin{array}{l}\text { Pseudobombax longiflorum (Mart. \& } \\
\text { Zucc.) A. Robyns }\end{array}$ & Arv & 2 & $1.091,05$ \\
\hline \multicolumn{4}{|l|}{ LEGUMINOSAE } \\
\hline $\begin{array}{l}\text { Dimorphandra mollis Benth. } \\
\text { SAPINDACEAE }\end{array}$ & Arv & 52 & $1.027,89$ \\
\hline $\begin{array}{l}\text { Matayba guianensis Aubl. } \\
\text { LEGUMINOSAE }\end{array}$ & Arb & 555 & 773,77 \\
\hline $\begin{array}{l}\text { Dalbergia violacea (Jacq.) Hoffsgg. } \\
\text { CARYOCARACEAE }\end{array}$ & Arv & 17 & 565,99 \\
\hline $\begin{array}{l}\text { Caryocar brasiliense Cambess. } \\
\text { LEGUMINOSAE }\end{array}$ & Arv & 2 & 540,33 \\
\hline $\begin{array}{l}\text { Acosmium subelegans (Mohlenbr.) } \\
\text { Yakovlev }\end{array}$ & Arv & 37 & 491,66 \\
\hline VOCHYSIACEAE & & & \\
\hline $\begin{array}{l}\text { Qualea grandiflora Mart. } \\
\text { MYRTACEAE }\end{array}$ & Arv & 6 & 488,46 \\
\hline $\begin{array}{l}\text { Eugenia aurata O. Berg. } \\
\text { SOLANACEAE }\end{array}$ & Arb & 30 & 302,43 \\
\hline $\begin{array}{l}\text { SOLANACEAE } \\
\text { Solanum lycocarpum A. St.-Hil. }\end{array}$ & Arv & 23 & 298,35 \\
\hline ASTERACEAE & & & \\
\hline $\begin{array}{l}\text { Vernonia polyanthes Less. } \\
\text { MYRTACEAE }\end{array}$ & Arb & 129 & 282,83 \\
\hline $\begin{array}{l}\text { Eugenia punicifolia (Kunth) DC. } \\
\text { MALPIGHIACEAE }\end{array}$ & Arb & 8 & 254,33 \\
\hline $\begin{array}{l}\text { Byrsonima intermedia A. Juss. } \\
\text { MYRTACEAE }\end{array}$ & Arb & 101 & 240,65 \\
\hline $\begin{array}{l}\text { Myrcia variabilis DC. } \\
\text { HIPPOCRATEACEAE }\end{array}$ & Arb & 34 & 207,49 \\
\hline $\begin{array}{l}\text { Tontelea micrantha (Mart. ex Schult.) } \\
\text { A.C. Sm. }\end{array}$ & Arb & 95 & 184,58 \\
\hline ERYTHROXYLACEAE & & & \\
\hline $\begin{array}{l}\text { Erythroxylum daphnites Mart. } \\
\text { COMBRETACEAE }\end{array}$ & Arv & 7 & 155,78 \\
\hline $\begin{array}{l}\text { Terminalia argentea (Camb.) Mart. } \\
\text { NYCTAGINACEAE }\end{array}$ & Arv & 7 & 142,54 \\
\hline $\begin{array}{l}\text { Guapira noxia (Netto) Lundell } \\
\text { ERYTHROXYLACEAE }\end{array}$ & Arv & 12 & 135,31 \\
\hline $\begin{array}{l}\text { Erythroxylum campestre A. St.-Hil. } \\
\text { ASTERACEAE }\end{array}$ & Arb & 93 & 134,88 \\
\hline $\begin{array}{l}\text { Alomia fastigiata Benth. } \\
\text { OCHNACEAE }\end{array}$ & Arb & 74 & 129,19 \\
\hline $\begin{array}{l}\text { Ouratea spectabilis (Mart. ex Engl.) } \\
\text { Engl. }\end{array}$ & Arv & 6 & 127,70 \\
\hline MYRTACEAE & & & \\
\hline $\begin{array}{l}\text { Myrcia rostrata DC. } \\
\text { MYRTACEAE }\end{array}$ & Arb & 39 & 94,36 \\
\hline Eugenia calycina Cambess. & Arb & 37 & 88,61 \\
\hline
\end{tabular}

\begin{tabular}{|c|c|c|c|}
\hline Famílias/Espécies & $\mathrm{H}$ & $\mathrm{DA}$ & DoA \\
\hline \multicolumn{4}{|l|}{ FLACOURTIACEAE } \\
\hline $\begin{array}{l}\text { Casearia sylvestris Sw. } \\
\text { ERYTHROXYLACEAE }\end{array}$ & Arb & 54 & 88,10 \\
\hline $\begin{array}{l}\text { Erythroxylum tortuosum Mart. } \\
\text { MALPIGHIACEAE }\end{array}$ & Arb & 15 & 86,84 \\
\hline $\begin{array}{l}\text { Byrsonima verbascifolia (L.) DC. } \\
\text { MELASTOMATACEAE }\end{array}$ & Arv & 3 & 83,36 \\
\hline $\begin{array}{l}\text { Miconia albicans (Sw.) Triana } \\
\text { MALPIGHIACEAE }\end{array}$ & Arb & 9 & 83,26 \\
\hline $\begin{array}{l}\text { Byrsonima coccolobaefolia Kunth } \\
\text { NYCTAGINACEAE }\end{array}$ & Arv & 2 & 78,79 \\
\hline $\begin{array}{l}\text { Guapira graciliflora (Schmidt) Lundell } \\
\text { LEGUMINOSAE }\end{array}$ & Arv & 3 & 78,01 \\
\hline $\begin{array}{l}\text { Senna rugosa (G. Don) H.S. Irwin \& } \\
\text { Barneby }\end{array}$ & Arb & 59 & 77,79 \\
\hline MALPIGHIACEAE & & & \\
\hline $\begin{array}{l}\text { Byrsonima crassa Nied. } \\
\text { SMILACACEAE }\end{array}$ & Arv & 7 & 76,71 \\
\hline $\begin{array}{l}\text { Smilax brasiliensis Spreng. } \\
\text { MALPIGHIACEAE }\end{array}$ & Cip & 69 & 72,08 \\
\hline $\begin{array}{l}\text { Byrsonima basiloba A. Juss. } \\
\text { EUPHORBIACEAE }\end{array}$ & Arv & 4 & 71,72 \\
\hline $\begin{array}{l}\text { Maprounea guianensis Aubl. } \\
\text { MYRTACEAE }\end{array}$ & Arb & 30 & 69,36 \\
\hline $\begin{array}{l}\text { Campomanesia adamantium } \\
\text { (Cambess.) O. Berg. }\end{array}$ & Arb & 36 & 68,68 \\
\hline BIGNONIACEAE & & & \\
\hline $\begin{array}{l}\text { Jacaranda caroba (Vell.) A. DC. } \\
\text { MELASTOMATACEAE }\end{array}$ & Arb & 43 & 68,16 \\
\hline $\begin{array}{l}\text { Leandra lacunosa Cogn. } \\
\text { MYRSINACEAE }\end{array}$ & Arb & 15 & 65,27 \\
\hline $\begin{array}{l}\text { Myrsine guianensis (Aubl.) Kuntze } \\
\text { SYMPLOCACEAE }\end{array}$ & Arv & 6 & 62,50 \\
\hline $\begin{array}{l}\text { Symplocos platyphylla (Pohl) Benth. } \\
\text { MELASTOMATACEAE }\end{array}$ & Arv & 9 & 61,07 \\
\hline $\begin{array}{l}\text { Miconia stenostachya Schrank \& } \\
\text { Mart. ex DC. }\end{array}$ & Arb & 17 & 60,75 \\
\hline RUBIACEAE & & & \\
\hline $\begin{array}{l}\text { Tocoyena formosa (Cham. \& Schltdl.) } \\
\text { K. Schum. } \\
\text { MYRTACEAE }\end{array}$ & Arb & 12 & 47,53 \\
\hline $\begin{array}{l}\text { Myrcia tomentosa (Aubl.) DC. } \\
\text { LAURACEAE }\end{array}$ & Arv & 8 & 44,90 \\
\hline $\begin{array}{l}\text { Ocotea minarum (Ness \& C. Mart.) Mez } \\
\text { RUBIACEAE }\end{array}$ & Arv & 5 & 44,80 \\
\hline $\begin{array}{l}\text { Palicourea rigida Kunth } \\
\text { NYCTAGINACEAE }\end{array}$ & Arb & 8 & 41,13 \\
\hline $\begin{array}{l}\text { Neea theifera } \text { Orsted } \\
\text { STYRACACEAE }\end{array}$ & Arv & 2 & 39,12 \\
\hline $\begin{array}{l}\text { Styrax ferrugineus Nees \& Mart. } \\
\text { LEGUMINOSAE }\end{array}$ & Arv & 13 & 38,36 \\
\hline $\begin{array}{l}\text { Inga laurina (Sw.) Willd. } \\
\text { PROTEACEAE }\end{array}$ & Arv & 3 & 36,53 \\
\hline Roupala montana Aubl. & Arv & 6 & 35,86 \\
\hline
\end{tabular}


14 Resende, Araújo, Oliveira, Oliveira, Ávila Júnior: A comunidade vegetal e as características abióticas de um campo...

Tabela 2 (continuação)

\begin{tabular}{|c|c|c|c|}
\hline Famílias/Espécies & $\mathrm{H}$ & DA & DoA \\
\hline \multicolumn{4}{|l|}{ DILLENIACEAE } \\
\hline $\begin{array}{l}\text { Davilla elliptica A. St.-Hil. } \\
\text { RUTACEAE }\end{array}$ & Arb & 29 & 35,21 \\
\hline $\begin{array}{l}\text { Hortia brasiliensis } \text { Vand. } \\
\text { VOCHYSIACEAE }\end{array}$ & Arb & 12 & 33,73 \\
\hline $\begin{array}{l}\text { Qualea multiflora Mart. } \\
\text { MYRTACEAE }\end{array}$ & Arv & 7 & 33,43 \\
\hline $\begin{array}{l}\text { Campomanesia pubescens (DC.) } \\
\text { O. Berg. }\end{array}$ & Arb & 24 & 32,19 \\
\hline ASTERACEAE & & & \\
\hline $\begin{array}{l}\text { Vernonia ferruginea Less. } \\
\text { BIGNONIACEAE }\end{array}$ & Arb & 8 & 30,79 \\
\hline $\begin{array}{l}\text { Tabebuia ochracea (Cham.) Standl. } \\
\text { MELIACEAE }\end{array}$ & Arv & 21 & 30,25 \\
\hline $\begin{array}{l}\text { Cabralea canjerana subsp. Polytricha } \\
\text { (A. Juss.) T.D. Penn. }\end{array}$ & Arb & 7 & 30,14 \\
\hline EGUMINOSAE & & & \\
\hline $\begin{array}{l}\text { Bowdichia virgilioides Kunth } \\
\text { MYRTACEAE }\end{array}$ & Arv & 1 & 28,27 \\
\hline $\begin{array}{l}\text { Myrcia guianensis (Aubl.) DC. } \\
\text { ANNONACEAE }\end{array}$ & Arb & 25 & 27,39 \\
\hline $\begin{array}{l}\text { Annona dioica A. St.-Hil. } \\
\text { ERIOCAULACEAE }\end{array}$ & Arv & 18 & 27,22 \\
\hline $\begin{array}{l}\text { Paepalanthus speciosus Körn. } \\
\text { LEGUMINOSAE }\end{array}$ & Arb & 5 & 27,12 \\
\hline $\begin{array}{l}\text { Acosmium dasycarpum (Vog.) Yakov. } \\
\text { ASTERACEAE }\end{array}$ & Arv & 3 & 26,01 \\
\hline $\begin{array}{l}\text { Eupatorium ferrugineum } \text { Gardner } \\
\text { CONNARACEAE }\end{array}$ & Arb & 18 & 25,45 \\
\hline $\begin{array}{l}\text { Rourea induta } \text { Planch. } \\
\text { LAURACEAE }\end{array}$ & Arb & 23 & 24,86 \\
\hline $\begin{array}{l}\text { Ocotea corymbosa (Meisn.) Mez } \\
\text { LYTHRACEAE }\end{array}$ & Arv & 3 & 24,72 \\
\hline $\begin{array}{l}\text { Diplusodon virgatus Pohl } \\
\text { RUBIACEAE }\end{array}$ & Arb & 9 & 24,24 \\
\hline $\begin{array}{l}\text { Rudgea viburnoides (Cham.) Benth. } \\
\text { ANNONACEAE }\end{array}$ & Arb & 6 & 22,90 \\
\hline $\begin{array}{l}\text { Annona coriacea } \text { Mart. } \\
\text { LEGUMINOSAE }\end{array}$ & Arv & 3 & 22,38 \\
\hline $\begin{array}{l}\text { Bauhinia holophylla (Bong.) Steud. } \\
\text { ERYTHROXYLACEAE }\end{array}$ & Arb & 21 & 22,05 \\
\hline $\begin{array}{l}\text { Erythroxylum deciduum A. St.-Hil. } \\
\text { LEGUMINOSAE }\end{array}$ & Arb & 11 & 21,14 \\
\hline $\begin{array}{l}\text { Machaerium opacum Vog. } \\
\text { MALVACEAE }\end{array}$ & Arv & 4 & 20,90 \\
\hline $\begin{array}{l}\text { Sida cordifolia } \mathrm{L} \text {. } \\
\text { EBENACEAE }\end{array}$ & Arb & 21 & 20,49 \\
\hline $\begin{array}{l}\text { Diospyros burchellii DC. } \\
\text { MYRTACEAE }\end{array}$ & Arv & 4 & 18,08 \\
\hline $\begin{array}{l}\text { Eugenia livida O. Berg. } \\
\text { RUBIACEAE }\end{array}$ & Arb & 4 & 14,96 \\
\hline $\begin{array}{l}\text { Alibertia obtusa K. Schum. } \\
\text { MALPIGHIACEAE }\end{array}$ & Arb & 9 & 14,63 \\
\hline $\begin{array}{l}\text { Heteropteris sp. } 1 \\
\text { IALPIGHIACEAE }\end{array}$ & Arb & 13 & 12,53 \\
\hline $\begin{array}{l}\text { Banisteriopsis sp. } 2 \\
\text { IPPOCRATEACEAE }\end{array}$ & Arb & 9 & 12,02 \\
\hline Peritassa campestris (Cam & $\mathrm{Arb}$ & 7 & 11,23 \\
\hline
\end{tabular}

\begin{tabular}{|c|c|c|c|}
\hline Famílias/Espécies & $\mathrm{H}$ & DA & DoA \\
\hline \multicolumn{4}{|l|}{ CELASTRACEAE } \\
\hline $\begin{array}{l}\text { Austroplenckia populnea (Reissek) } \\
\text { Lundell }\end{array}$ & Arv & 2 & 10,56 \\
\hline \multicolumn{4}{|l|}{ MELASTOMATACEAE } \\
\hline \multicolumn{4}{|l|}{ ASTERACEAE } \\
\hline Eupatorium sp. & Arb & 9 & 8,49 \\
\hline \multicolumn{4}{|l|}{ MALPIGHIACEAE } \\
\hline $\begin{array}{l}\text { Peixotoa tomentosa A. Juss. } \\
\text { RUBIACEAE }\end{array}$ & Arb & 4 & 8,18 \\
\hline $\begin{array}{l}\text { Coussarea hydrangeifolia (Benth.) } \\
\text { Müll. Arg. }\end{array}$ & Arv & 1 & 8,04 \\
\hline \multicolumn{4}{|l|}{ MYRTACEAE } \\
\hline $\begin{array}{l}\text { Eugenia paranahybensis O. Berg. } \\
\text { MYRTACEAE }\end{array}$ & Arb & 4 & 7,94 \\
\hline $\begin{array}{l}\text { Myrcia uberavensis } \mathrm{O} . \text { Berg. } \\
\text { ANNONACEAE }\end{array}$ & Arb & 11 & 6,63 \\
\hline $\begin{array}{l}\text { Annona cornifolia A. St.Hil. } \\
\text { CECROPIACEAF }\end{array}$ & Arb & 4 & 5,88 \\
\hline Cecropia pachystachya Trécul & Arv & 2 & 5,69 \\
\hline \multicolumn{4}{|l|}{ OCHNACEAE } \\
\hline $\begin{array}{l}\text { Ouratea } \mathrm{sp} \text {. } \\
\text { SOLANACEAE }\end{array}$ & Arb & 4 & 5,29 \\
\hline Cestrum sp. & Arb & 5 & 5,02 \\
\hline \multicolumn{4}{|l|}{ VERBENACEAE } \\
\hline \multicolumn{4}{|l|}{ ASTERACEAE } \\
\hline Vernonia sp. 1 & Arb & 4 & 4,69 \\
\hline \multicolumn{4}{|l|}{ MALPIGHIACEAE } \\
\hline Banisteriopsis sp. 1 & Arb & 2 & 4,47 \\
\hline \multicolumn{4}{|l|}{ MYRTACEAE } \\
\hline \multicolumn{3}{|l|}{ MYRTACEAE } & 4,38 \\
\hline \multicolumn{4}{|l|}{ STERCULIACEAE } \\
\hline Waltheria sp. & Arb & 7 & 3,71 \\
\hline \multicolumn{4}{|l|}{ MELASTOMATACEAE } \\
\hline \multicolumn{4}{|l|}{ LEGUMINOSAE } \\
\hline $\begin{array}{l}\text { Andira humilis Mart. ex Benth. } \\
\text { FLACOURTIACEAE }\end{array}$ & Arb & 1 & 2,84 \\
\hline $\begin{array}{l}\text { Casearia grandiflora Cambess. } \\
\text { LEGUMINOSAE }\end{array}$ & Arv & 2 & 2,77 \\
\hline $\begin{array}{l}\text { Stryphnodendron polyphyllum Mart. } \\
\text { RUBIACEAE }\end{array}$ & Arv & 4 & 2,74 \\
\hline $\begin{array}{l}\text { Declieuxia fruticosa (Willd. ex Roem. } \\
\text { \& Schult.) Kuntze }\end{array}$ & Arb & 3 & 2,55 \\
\hline MYRTACEAE & & & \\
\hline $\begin{array}{l}\text { Myrcia rhodeosepala Kiaersk. } \\
\text { MYRTACEAE }\end{array}$ & Arb & 3 & 2,02 \\
\hline Psidium firmum $\mathrm{O}$. Berg & Arv & 1 & 2,01 \\
\hline ASTERACEAE & & & \\
\hline Vernonia sp. 2 & Arb & 2 & 1,82 \\
\hline MYRTACEAE & & & \\
\hline $\begin{array}{l}\text { Myrcia bella Cambess. } \\
\text { ASTERACEAF }\end{array}$ & Arb & 1 & 1,76 \\
\hline Gochnatia paniculata (Less.) Cabrera & Arb & 2 & $\begin{array}{r}1,74 \\
\text { ntinua }\end{array}$ \\
\hline
\end{tabular}


Tabela 2 (continuação)

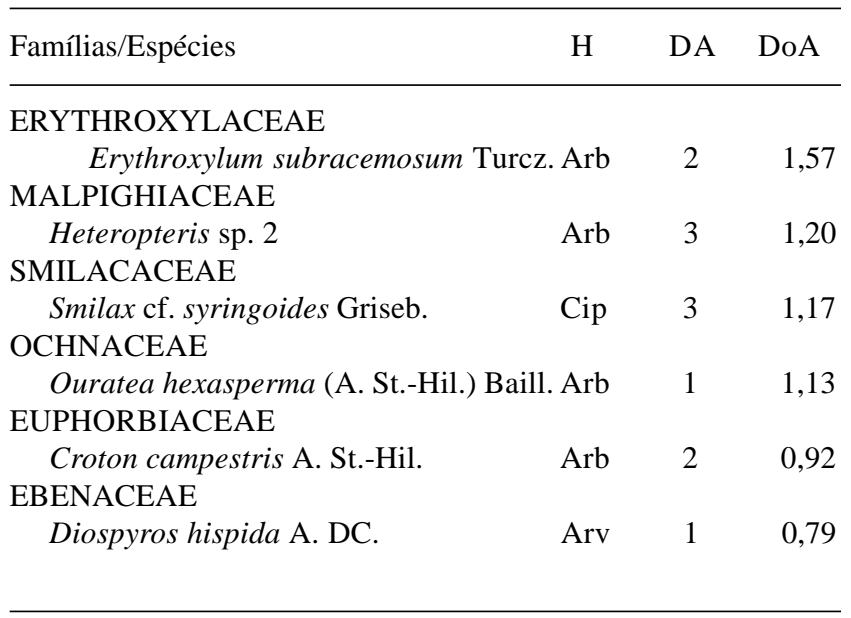

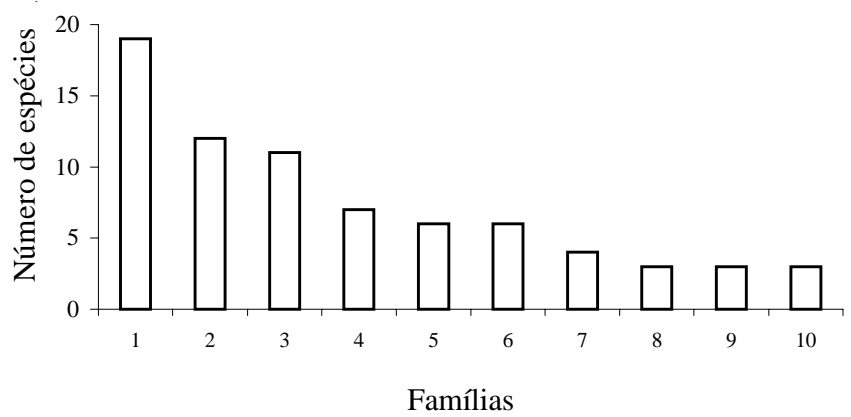

Figura 5. As dez famílias com maior número de espécies amostradas em um campo de murundu em Uberlândia, MG. 1 = Myrtaceae, 2 = Leguminosae, 3 = Malpighiaceae, 4 = Asteraceae, 5 = Erythroxylaceae, $6=$ Rubiaceae, $7=$ Melastomataceae, $8=$ Annonaceae, $9=$ Nictaginaceae, $10=$ Ochnaceae .

$(6,4 \%$ e 10 a $20 \%$, respectivamente). Os maiores murundus foram encontrados por Oliveira-Filho (1992a) em local levemente inclinado, próximo à Cuiabá $\left(270,04 \mathrm{~m}^{2}\right.$ e $\left.109,82 \mathrm{~m}^{3}\right)$ e Diniz de Araújo Neto et al. (1986), em áreas de campo limpo no Distrito Federal $\left(141,53 \mathrm{~m}^{3}\right)$. O menor tamanho dos murundus da fazenda Munjolinho deve-se, possivelmente, ao pisoteio do gado bovino e à erosão pluvial.

Os murundus do presente estudo, com maior volume de solo, parecem propiciar a ocorrência de algumas espécies lenhosas do Cerrado, que, conforme Diniz de Araújo Neto et al. (1986), Oliveira-Filho (1992b) e Ponce \& Cunha (1993), não sobrevivem em locais alagados. Lobo \& Joly (1998) afirmam que a saturação hídrica no solo pode ter caráter seletivo, resultando em diferenças florísticas entre comunidades vegetais. Nesse sentido, Joly \& Crawford (1982) demonstraram que algumas espécies de Cerrado não são capazes de tolerar solos hidromórficos e, por isso, nos campos de murundu, ocorrem somente sobre os morrotes.

\begin{tabular}{|c|c|c|c|}
\hline Famílias/Espécies & $\mathrm{H}$ & DA & DoA \\
\hline \multicolumn{4}{|l|}{ LEGUMINOSAE } \\
\hline $\begin{array}{l}\text { Stryphnodendron adstringens (Mart.) } \\
\text { Coville }\end{array}$ & Arv & 1 & 0,50 \\
\hline \multicolumn{4}{|l|}{ BURSERACEAE } \\
\hline $\begin{array}{l}\text { Protium ovatum Engl. } \\
\text { APOCYNACEAE }\end{array}$ & Arb & 1 & 0,50 \\
\hline $\begin{array}{l}\text { Aspidosperma tomentosum Mart. } \\
\text { STERCULIACEAE }\end{array}$ & Arv & 1 & 0,39 \\
\hline $\begin{array}{l}\text { Helicteres brevispira A. St.-Hil. } \\
\text { LAMIACEAE }\end{array}$ & Arb & 1 & 0,38 \\
\hline $\begin{array}{l}\text { Hyptis sp. } \\
42 \text { famílias }\end{array}$ & Arb & 1 & 0,28 \\
\hline 116 espécies & & 2.470 & $14.878,25$ \\
\hline
\end{tabular}

O solo do campo de murundu estudado, segundo os critérios de Lopes \& Cox (1977) para ambientes de Cerrado, é considerado como de alto teor de $\mathrm{Al}$ e de acidez, com baixa disponibilidade de bases trocáveis. Solos com tais características foram encontrados sob vegetação de cerrado no Triângulo Mineiro (Goodland 1979) e no município de Uberlândia (Costa \& Araújo, 2001). Oliveira-Filho (1992a) encontrou solos sob murundus com propriedades químicas semelhantes aos cupinzeiros próximos. $\mathrm{Na}$ área estudada, a pequena diferença na disponibilidade de nutrientes entre os solos dos murundus e a área plana adjacente pode ter sido resultante de processos erosivos sobre os morrotes.

As características do solo dos murundus com volume superior a $1 \mathrm{~m}^{3}$ foram favoráveis à ocupação dos mesmos por espécies lenhosas, principalmente da flora dos cerrados vizinhos. Das 116 espécies amostradas no presente estudo, 50 foram similares às arbóreas de Cerrado estudadas por Araújo et al. (1997) no município de Uberlândia e grande parte das arbustivas ocorre em cerrados mais abertos do Triângulo Mineiro (Goodland, 1979). Isso indica que a maioria da vegetação lenhosa dos campos de murundus de Uberlândia é de Cerrado, característica também encontrada por Oliveira-Filho (1992b) nesse mesmo ambiente na Baixada Cuiabana.

Entretanto, a similaridade florística (Sørensen) entre o campo de murundu da Baixada Cuiabana (OliveiraFilho 1992b) e a do presente estudo foi de apenas $11 \%$, embora entre os cerrados das duas regiões tenha sido de $49,7 \%$. Isso mostra que, embora sejam ocupadas por vegetação de cerrado, diferenças geográficas, como havia sido discutido por Ratter et al. (1996), possibilitam que espécies diferentes ocorram em cada local. Curatella americana, a espécie mais importante em 
murundus do Pantanal, não ocorreu na área estudada, apesar de ter sido amostrada em cerrados do município de Uberlândia por Araújo et al. (1997). Segundo Oliveira-Filho (1992b) e Eiten (1985), esta é uma das espécies mais tolerantes à variação sazonal na saturação hídrica do solo.

No presente estudo não foram encontradas espécies exclusivas, a exemplo do que ocorreu com Annona pygmaea nos murundus da Baixada Cuiabana (Oliveira-Filho 1992b). Por outro lado, as espécies com alta densidade comuns a esses dois locais foram Matayba guianensis e Erythroxylum suberosum. Segundo Araújo et al. (1997), M. guianensis, com hábito arbustivo, apresenta grande população em cerrados alterados no município de Uberlândia. E. suberosum possui maior número de indivíduos em cerrados abertos (Goodland 1979). Essas espécies parecem ser competitivas tanto em solos mais secos do cerrado quanto em locais com maior saturação de água no período das chuvas, como acontece nos murundus.

No entanto, as plantas que se destacam na paisagem, ocupando os murundus, são árvores dominantes como Copaifera langsdorffii, Pseudobombax longiflorum e Caryocar brasiliense. Estas espécies apresentaram baixas densidades, porém as maiores áreas basais. C. langsdorffii é conhecida por sua ampla distribuição tanto em florestas (Araújo \& Haridasan 1997; Schiavini 1992) quanto em cerrados com maior cobertura arbórea (Araújo et al. 1997; Costa \& Araújo 2001). Caryocar brasiliense é conhecida por sua dominância nos cerrados (Araújo et al. 1997; Ribeiro et al. 1985), enquanto Pseudobombax longiflorum ocorre em florestas e cerrados, porém com populações menores (Sampaio et al. 2000; Ribeiro et al. 1985).

Outras espécies arbóreas de floresta amostradas por Araújo \& Haridasan (1997), como Cecropia pachystachya, Ocotea corymbosa, O. minarum e Inga laurina, foram encontradas com indivíduos jovens. Tal fato pode ser indício de que o ambiente também é propício para a ocupação de espécies florestais, pelo menos em seus estádios iniciais de desenvolvimento.

A maior diversidade encontrada na área estudada, em relação aos dados obtidos por Oliveira-Filho (1992b) pode ser resultante da heterogeneidade ambiental provocada pela antropização, como pisoteio, pastejo bovino e queimadas. Esses fatores podem ter favorecido a ocupação de espécies resistentes a esses impactos. Áreas protegidas tendem a apresentar menor riqueza que áreas pastejadas correspondentes (Pandey \& Singh 1991; Belsky 1992; Guimarães et al. 2002). Mas a alteração da riqueza de espécies está diretamente relacionada à intensidade do pastejo (Milchunas et al. 1988). No que diz respeito ao fogo, tal fato é evidenciado pela presença de Dimorphandra mollis, Rourea induta, Caryocar brasiliense, Byrsonima verbascifolia, Neea theifera e Ouratea hexasperma, espécies resistentes a queimadas, conforme observado por Sato \& Miranda (1996) e Silva et al. (1996). Dessa forma, elevados níveis de diversidade de espécies são mantidos em uma freqüência ou intensidade "intermediária" de perturbação (Huston 1994).

\section{Agradecimentos}

Os Autores agradecem a Ana Flávia Alves Parente, Cláudia Ribeiro Borges Silva, Elisa Queiroz Garcia, Ludmila Mendes de Oliveira, Paulo José de Souza, Ricardo Alvarenga Ribeiro, Shirleny Romualdo Cardoso, Tânia Dolores, Welington Nunes da Silva e Wilton Pereira pelo auxílio na coleta de dados no campo; ao Dr. Christian Westerkamp, pelo auxílio com o Abstract; à Dra. Adriana Assis Arantes, pela identificação das espécies de Myrtaceae.

\section{Referências bibliográficas}

Araújo, G. M. \& Haridasan, M. 1997. Estrutura fitossociológica de duas matas mesófilas semidecíduas, em Uberlândia, Triângulo Mineiro. Naturalia 22(1): 115-129.

Araújo, G. M.; Nunes, J. J. N.; Rosa, A. G. \& Resende, E. R. 1997. Estrutura comunitária de vinte áreas de cerrados residuais no município de Uberlândia, MG. Daphne 7(2): 7-14.

Belsky, A. J. 1992. Effects of grazing, competition, disturbance, and fire on species composition and diversity in grassland communities. Journal of Vegetation Science 3: $187-200$.

Bernacci, L. C. \& Leitão Filho, H. F. 1996. Flora fanerogâmica da floresta da Fazenda São Vicente, Campinas, São Paulo. Revista Brasileira de Botânica 19(2): 149-164.

Cain, S. A. \& Castro, G. M. O. 1959. Manual of vegetation analysis. Harper, New York.

Costa, A. A. \& Araújo, G. M. 2001. Comparação da vegetação arbórea de cerradão e de cerrado na reserva do Panga, Uberlândia, Minas Gerais. Acta Botanica Brasilica 15(1): 63-72.

Diniz de Araújo Neto, M.; Furley, P. A.; Haridasan, M. \& Johnson, C. E. 1986. The murundus of the cerrado region of Central Brazil. Journa l of Tropical Ecology 2: 17-35.

Eiten, G. 1972. The cerrado vegetation of Brazil. Botanical Review 38(2): 201-341.

Eiten, G. 1985. Vegetation near Santa Teresinha, NE Mato Grosso. Acta Amazônica 15(3/4): 275-301. 
Eiten, G. 1990. Vegetação do Cerrado. Pp. 9-65. In: M. N. Pinto (coord.). Cerrado: caracterização, ocupação e perspectives. SEMATEC, UnB, Brasília.

EMBRAPA. 1979. Serviço nacional de levantamento e conservação dos solos. Manual de métodos de análise do solo, Rio de Janeiro.

Furley, P. A. 1986. Classification and distribution of murundus in the cerrado of Central Brazil. Journal of Biogeography 13: $265-268$.

Furley, P. A. \& Ratter, J. A. 1988. Soil resources and plant communities of the central Brazilian cerrado and their development. Journal of Biogeography 15: 97-108.

Goodland, R. 1979. Análise ecológica da vegetação do cerrado. Pp. 61-174. In: R. Goodland \& M. G. Ferri (eds.). Ecologia do cerrado. EDUSP, São Paulo.

Guimarães, A. J. M.; Araújo, G. M. \& Corrêa, G. F. 2002. Estrutura fitossociológica em área natural e antropizada de uma vereda em Uberlândia, MG. Acta Botanica Brasilica 16: 317-329.

Huston, M. A. 1994. Biological Diversity - the coexistence of species on changing landscapes. Cambridge University Press, Cambridge.

Joly, C. A. \& Crawford, M. M. 1982. Variation in tolerance and metabolic responses to flooding in some tropical trees. Journal of Experimental Botany 33: 799-809.

Lobo, P. C. \& Joly, C. A. 1998. Tolerance to hypoxia and anoxia in neotropical tree species. Pp. 137-156. In: F. R. Scarano \& A. C. Franco (eds.). Ecophysiological strategies of xerophytic and amphibious plants in the neotropics. Series Oecologia Brasiliensis IV. PPGEUFRJ, RJ.

Lopes, A. S. \& Cox, F. R. 1977. A survey of the fertility status of surface soils under "cerrado" vegetation in Brazil. Soil Science of Socciety America Journal 41(4): 742-747.

Matteucci, S. D. \& Colma, A. 1982. Metodologia para el estudio de la vegetação. $1^{\mathrm{a}}$ ed. Organization of Americam State, Washington.

Milchunas, D. G.; Sala, O. E. \& Lavenroth, W. K. 1988. A generalized model of the effects of grazing by large herbivores on grassland community structure. American Naturalisty 132: 87-106.

Oliveira-Filho, A. T. 1992a. Floodplain 'murundus' of Central Brazil: evidence for the termite-origin hypothesis. Journal of Tropical Ecology 8: 1-19.

Oliveira-Filho, A. T. 1992b. The vegetation of Brazilian 'murundus' - the island-effect on the plant community. Journal of Tropical Ecology 8: 465-486.
Oliveira-Filho, A. T. \& Furley, P. A. 1990. Monchão, cocuruto, murundu. Ciência Hoje 11: 30-37.

Pandey, C. B. \& Singh, J. S. 1991. Influence of grazing and soil conditions on secondary savanna vegetation in India. Journal of Vegetation Science 2: 95-102.

Ponce, V. M. \& Cunha, C. N. 1993. Vegetated earthmounds in tropical savannas of Central Brazil: a synthesis. Journal of Biogeography 20: 219-225.

Ratter, J. A.; Bridgewater, S.; Atkinson, R. \& Ribeiro, J. F. 1996. Analisys of the floristic composition of the Braziliam cerrado vegetation II: Comparison of the woody vegetation of 98 areas. Edinburg Journal of Botany 53(2): 153-180.

Ribeiro, J. F.; Silva, J. C. S. \& Batmanian, G. J. 1985. Fitossociologia de tipos fisionômicos de cerrado em Planaltina, DF. Revista Brasileira de Botânica 8(2): 131-142.

Rizzini, C. T. \& Rizzini, C. M. 1983. Dicionário botânico clássico latino-português abonado. IBDF - Jardim Botânico, Rio de Janeiro.

Rosa, R.; Lima, S. C. C. \& Assunção, W. L. 1991. Abordagem preliminar das condições climáticas de Uberlândia (MG). Sociedade e Natureza 3(5 e 6): 91-108.

Sampaio, A. B.; Walter, B. M. T. \& Felfili, J. M. 2000. Diversidade e distribuição de espécies arbóreas em duas Matas de Galeria na micro-bacia do Riacho Fundo, Distrito Federal. Acta Botanica Brasilica 14(2): 127-142.

Sato, M. N. \& Miranda, H. S. 1996. Mortalidade de plantas lenhosas do cerrado senso stricto submetidas a diferentes regimes de queima. Pp. 102-111. In: H. S. Miranda; C. H. Saito \& B. F. S. Dias (orgs.). Impactos de queimadas em áreas de cerrado e restinga. UnB, ECL, Brasília.

Schiavini, I. 1992. Estrutura de comunidades arbóreas de mata de galeria da Estação Ecológica do Panga (Uberlândia, MG). Tese de Doutorado. Universidade Estadual de Campinas, Campinas.

Schneider, M. O. \& Silva, D. B. 1991. Estrutura pedológica e dinâmica hídrica do "covoal" do Córrego da Fortaleza. Sociedade \& Natureza 3(5 e 6): 75-89.

Silva, G. T.; Sato, M. N. \& Miranda, H. S. 1996. Mortalidade de plantas lenhosas em um campo sujo de cerrado submetida a queimadas prescritas. Pp. 93-101. In: H. S. Miranda; C. H. Saito \& B. F. S. Dias (orgs.). Impactos de queimadas em áreas de cerrado e restinga. UnB, ECL, Brasília. 